\title{
The Multi-Track angiography catheter: a new tool for complex catheterisation in congenital heart disease
}

Philipp Bonhoeffer, Jean-Francois Piéchaud, Oliver Stümper, Damien Bonnet, Yacine Aggoun, Daniel Sidi, Jean Kachaner

\begin{abstract}
Objective-To develop a simple and versatile catheter system for complex cardiac catheterisation because angiography and pressure measurements during diagnostic and interventional cardiac catheterisation are often unsatisfactory.

Methods-The Multi-Track Angio catheter system is a single lumen side-hole catheter with a short distal extension containing a lumen for a standard guidewire. The catheter is introduced over a previously placed guidewire running through this distal extension. It can then be manipulated within the heart by sliding along the guidewire. The tip of the catheter is always stabilised by the guidewire. This stability enhances angiography and pressure recordings.

Results-The Multi-Track Angio catheter system was used in 84 patients (age 1 day-20 years). Thirty one procedures were diagnostic and 53 interventional. The decision to use the Multi-Track Angio catheter was based on three criteria: firstly, unsatisfactory angiography obtained with conventional equipment; secondly, difficult catheter course requiring use of a guidewire; and thirdly, requirement for angiography and pressure recordings during interventional procedures. No complications were encountered. High quality angiography could be performed in all cases without catheter recoil.
\end{abstract}

Conclusions-The Multi-Track Angio catheter system allows for high quality angiography and pressure recordings during diagnostic and interventional cardiac catheterisation. The advantage of the system is that both angiography and pressure recordings can be performed repeatedly from stable catheter positions using a previously placed guidewire. This reduces the need for guidewire manipulations or catheter exchanges and decreases procedure time and the risk of complications.

(Heart 1996;76:173-177)

Keywords: angiography; congenital heart disease; catheterisation
Diagnostic and interventional cardiac catheterisation are both often complex in patients with congenital heart disease. ${ }^{1}$ Difficulties are common because the catheters are seldom perfectly adapted to the morphology of the specific cardiac malformation. For these reasons, procedures are often protracted and in some cases studies are suboptimal. More versatile catheter equipment that can be adapted more easily to different body sizes and to various anatomical situations is required, particularly for interventional cardiology.

We designed a new catheter system ${ }^{2}$ which allows both high quality pressure measurements and angiography to be performed over a previously positioned guidewire. Such a system seems to improve catheter stability and reduce guidewire manipulation. We report our experience with the Multi-Track Angio catheter system in congenital heart disease.

\section{Methods}

DESCRIPTION OF THE MULTI-TRACK ANGIO CATHETER

The Multi-Track Angio catheter system (MAP Medical, North Lawrence, NY 12967) is a single lumen side-hole catheter with a short distal extension that contains a lumen for a standard guidewire (fig 1). We have used three sizes $(3 \cdot 5,4,5 \mathrm{~F})$. The catheter is introduced over a previously placed guidewire running through the distal extension. The introducer sheath must be large enough $(5,6,7$ $F$ respectively) to introduce the catheter sideby-side with the guidewire (table).

HANDLING OF THE MULTI-TRACK ANGIO CATHETER

Once a standard guidewire is securely placed within the distal region of the cardiac chamber

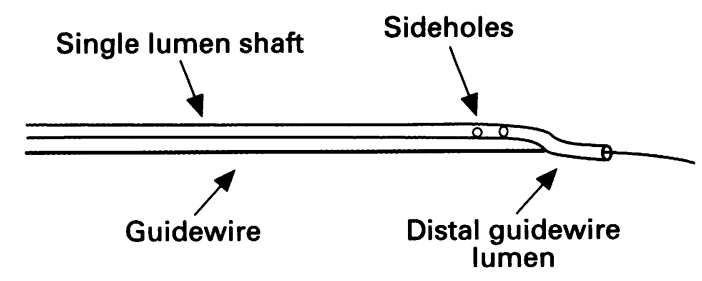

Figure 1 The Multi-Track Angio catheter is connected via the distal guidewire lumen to a previously positioned wire and is then advanced to the desired position. 
Technical characteristics

\begin{tabular}{lllc}
\hline Size & Guidewire (inch) & Introducer & Flow rate $(\mathrm{ml} / \mathrm{s})$ \\
\hline $3.5 \mathrm{~F}$ & 0.025 & $5 \mathrm{~F}$ & 6 \\
$4 \mathrm{~F}$ & 0.035 & $6 \mathrm{~F}$ & 10 \\
$5 \mathrm{~F}$ & 0.038 & $7 \mathrm{~F}$ & 15 \\
\hline
\end{tabular}

or vessel to be investigated (that is, the distal pulmonary artery) the end hole catheter is exchanged for the Multi-Track Angio catheter. The Multi-Track Angio catheter can be advanced with ease to the particular region of interest by maintaining slight tension on the guidewire, which in fact serves as a rail. Continuous pressure recordings can be obtained during catheter advancement or pullback. If it is difficult to advance the catheter, the shaft of the Multi-Track catheter can be stiffened with a guidewire introduced through the angiographic lumen or by looping the catheter, even if the guidewire serving as a rail takes a more direct route (fig 2). Also, during pullback recordings across tight stenoses it is often helpful to pullback on both the catheter and the guidewire simultaneously over a short distance. The tracking ability of the system can be further enhanced by independent manipulations of the guidewire and the catheter.

If necessary, the Multi-Track catheter can be disconnected from the guidewire simply by pushing its tip beyond the distal end of the

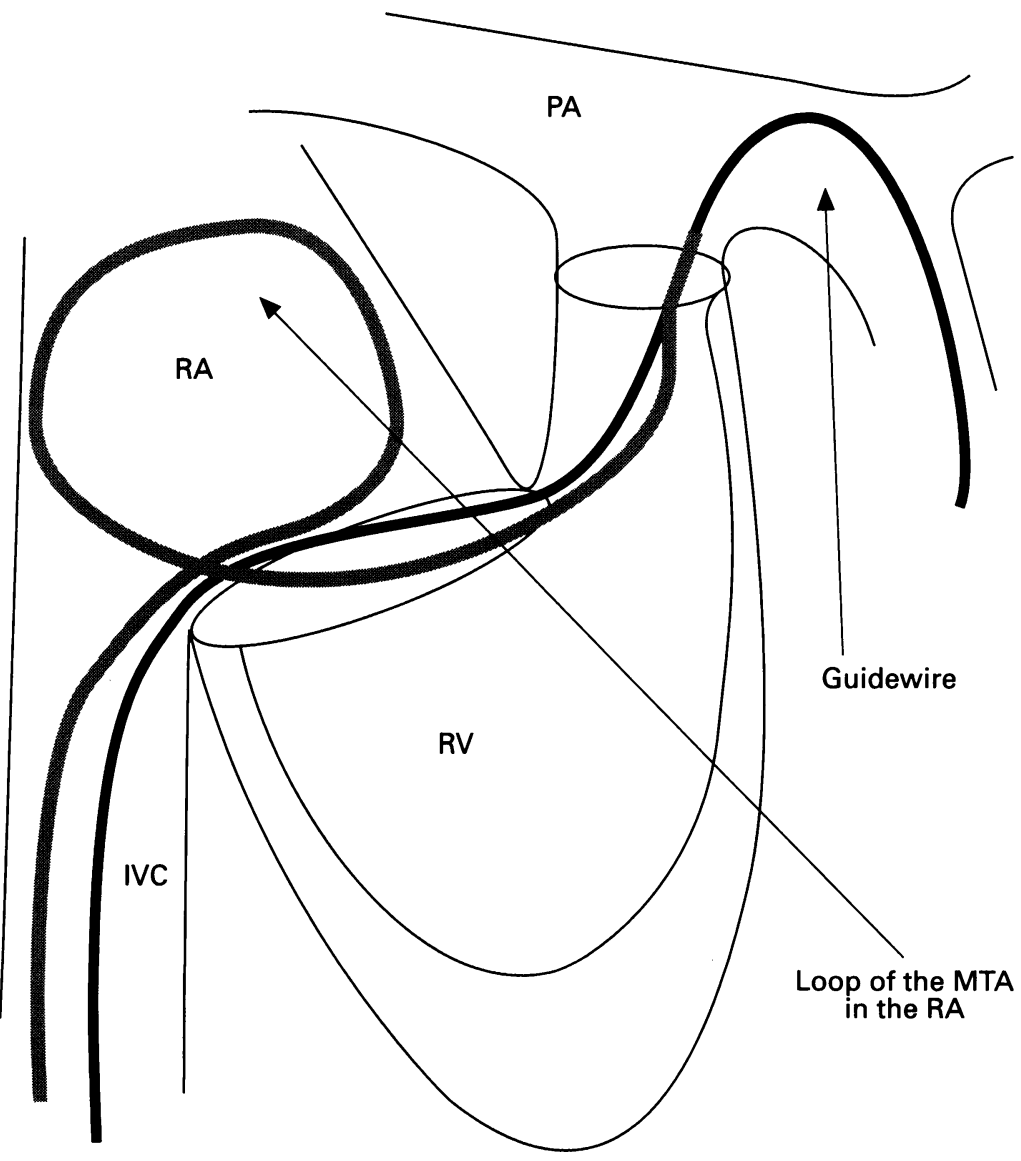

Figure 2 Advancement of the Multi-Track Angio catheter can sometimes be facilitated by looping the catheter even if the guidewire takes a more direct route. In this diagram the catheter is advanced into the pulmonary artery with a loop in the right atrium (see text). $P A$, pulmonary artery; $R A$, right atrium; $R V$, right ventricle; IVC, inferiour vena cava. guidewire. The catheter and the guidewire can then be pulled back independently.

\section{Results}

Eighty four patients with congenital heart disease underwent diagnostic or interventional catheterisation with the Multi-Track Angio catheter system at our institution. Their ages ranged from 1 day to 20 years and they weighed from $2 \cdot 1$ to 80 kilograms.

\section{DIAGNOSTIC PROCEDURES}

Thirty one diagnostic catheterisations were carried out. The Multi-Track Angio system was used to obtain pressure recordings and to perform angiography in complex congenital heart diseases when correct positioning of a conventional angiographic catheter was impossible or unstable.

In five patients with pulmonary atresia, ventricular septal defect, and major aorto-pulmonary collateral arteries (MAPCAs) it was possible to catheterise selectively from a venous approach the "true" pulmonary arteries via the anastomosis between the MAPCAs and the branches of the pulmonary artery. Angiographic studies using the end hole catheter which was used for entering the MAPCAs were unsatisfactory and potentially hazardous. High quality angiograms were obtained in all cases with the Multi-Track Angio catheter.

In some patients the Multi-Track Angio catheter was used as the first choice-for example, in a case of azygos continuation of the inferior vena cava.

\section{INTERVENTIONAL PROCEDURES}

Twenty seven patients, including three with complex cardiac malformations, had balloon dilatation of the pulmonary valve. A guidewire was placed within the left pulmonary artery using an end-hole catheter. Subsequent pressure recordings were obtained using the MultiTrack Angio catheter and the angiogram was performed at the level of the pulmonary valve annulus. Balloon dilatation was carried out thereafter without any further guidewire manipulations. Similar techniques were used in 22 patients who underwent balloon angioplasty (pulmonary branch stenosis in 12, re-coarctation in seven, right ventricle to pulmonary artery conduit in two, and pulmonary vein stenosis in one). In all cases high quality pressure recordings could be obtained and angiograms could be performed at the exact level of obstruction without risk of catheter recoil. Post-dilatation angiograms and pressure recordings were largely facilitated using the same guidewire that was used to position the Multi-Track Angio catheter (fig 3A and B).

Finally, angiography within the patent arterial duct enhanced the display of ductal morphology in three children, thereby facilitating double umbrella occlusion without an arterial approach.

We did not encounter any complications related to the use of the Multi-Track Angio catheter. 

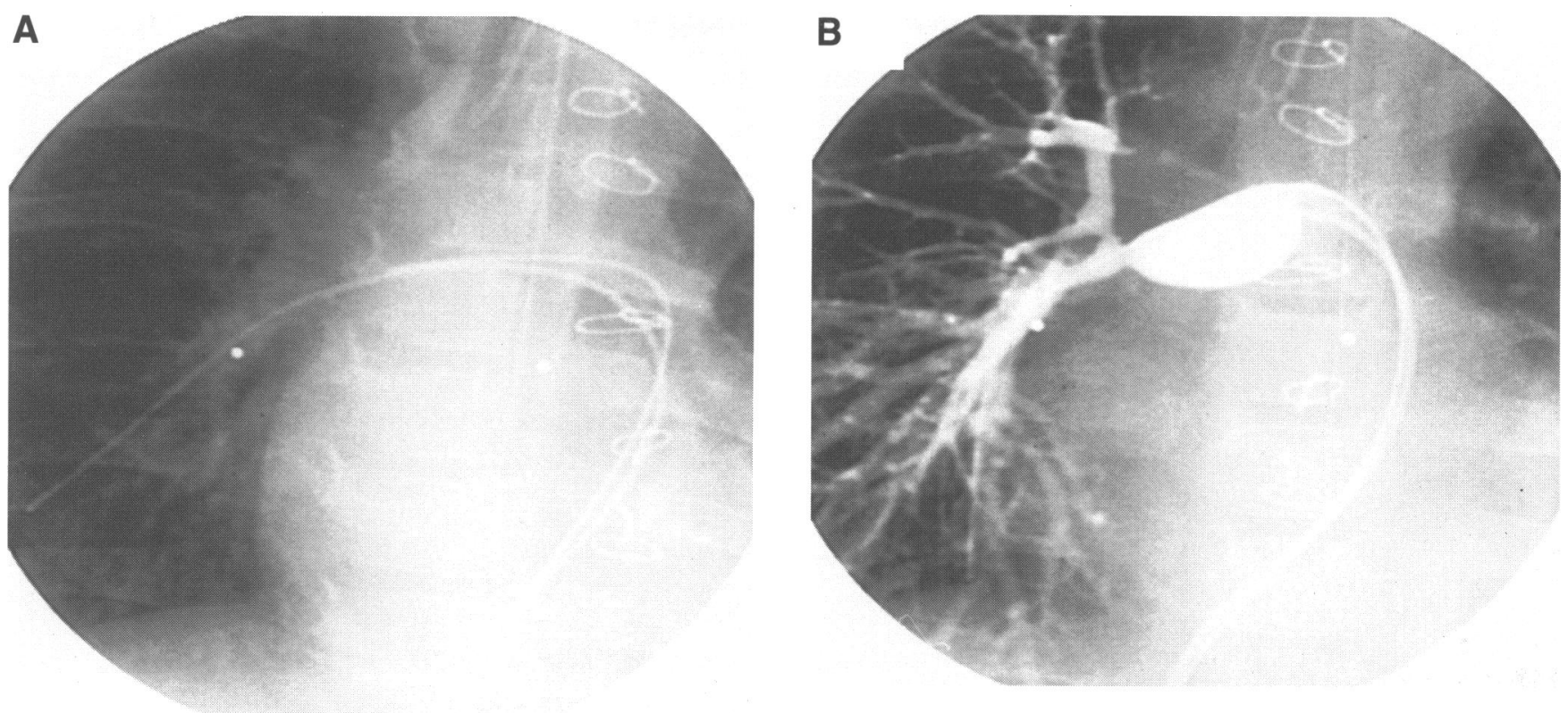

Figure 3 Patient with severe stenosis of the right pulmonary artery branch. The Multi-Track Angio catheter was advanced over the previously positioned guidewire (A). Angiography was done at the exact site of the pressure gradient (B).

\section{Discussion \\ BACKGROUND}

With the increasing complexity of diagnostic cardiac catheterisation and the developments in interventional cardiology in patients with congenital heart disease a wide variety of catheters have been designed. ${ }^{34}$ Nonetheless, in clinical practice complex catheterisation procedures are often hampered by the lack of perfectly adapted tools which help to solve the range of problems encountered. In our experience of more than 20000 cardiac catheterisations performed in children, one of the most

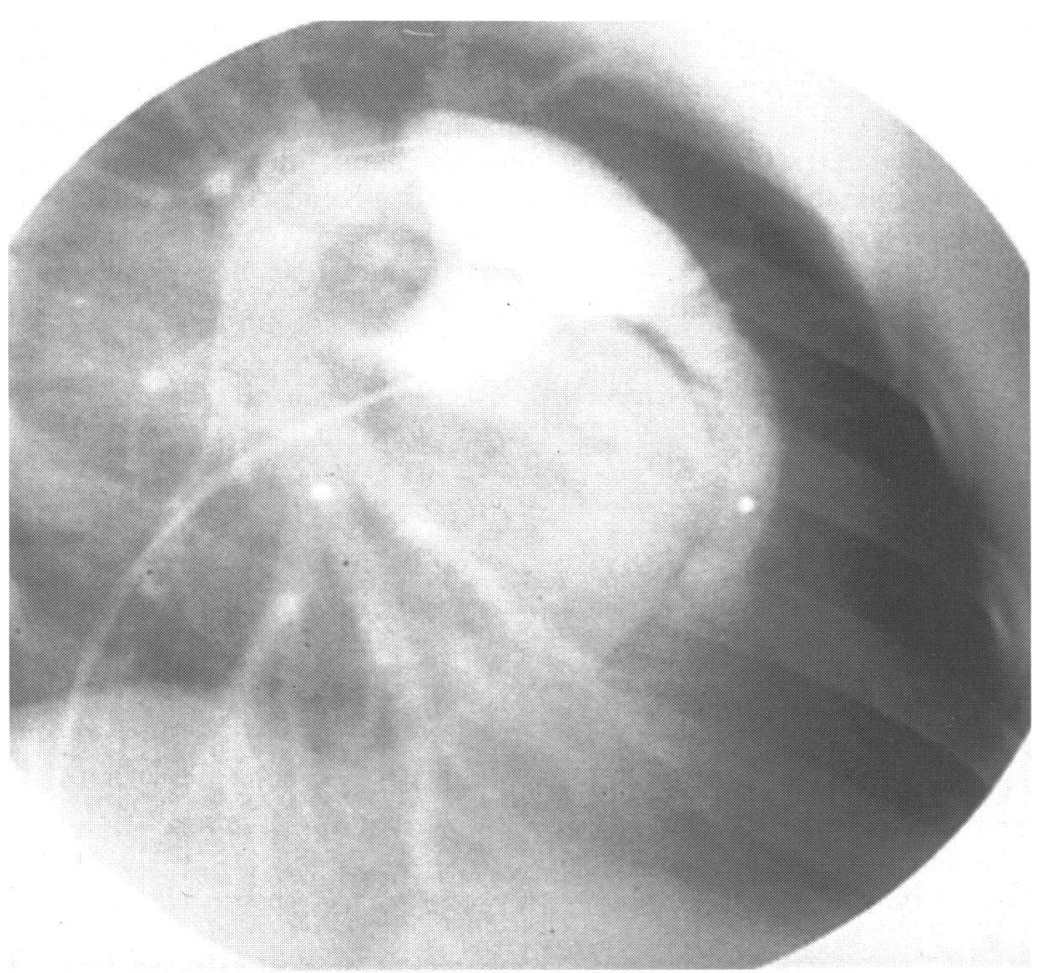

Figure 4 Angiogram in a case of a severe Ebstein's malformation. Despite the tiny right ventricle and the tricuspid valve regurgitation the Multi-Track Angio catheter stayed in a stable position during the injection of contrast medium. frequently encountered problems is instability of the catheter during angiography or pressure recordings, which leads to unsatisfactory results. Also, during interventional catheterisations, the need for frequent guidewire manipulations and catheter exchanges increases procedure time and adds to the potential risk. Finally, a new range of pathological conditions are encountered, in particular in adolescents and adults with congenital heart disease and in those patients who have undergone surgical repair. ${ }^{5-7}$ In view of the vast heterogenicity of patients with congenital heart disease coming forward for cardiac catheterisation, it is unlikely that a specifically designed catheter would be ideally suited to perform all these complex investigations. Therefore we set out to develop a new simple and versatile catheter system that can be used effectively in very different situations.

\section{DIAGNOSITIC CARDIAC CATHETERISATION}

With its tip firmly secured to a previously placed guidewire, the Multi-Track Angio catheter system allowed high quality angiography and pressure recordings to be obtained from extremely stable catheter positions (fig 3 ). Flow rate characteristics are similar to conventional angiographic catheters of comparable size.

Use the Multi-Track Angio system was indicated when it proved difficult to gain stable catheter positions with conventional equipment and when the catheter course was extremely difficult.

The major advantages of the Multi-Track Angio catheter during diagnostic cardiac catheterisation are that angiography can be performed from extremely stable positions (figs 4 and 5), thereby eliminating the risk of catheter recoil and intramyocardial injections, and that very controlled pressure pullback traces can be obtained for precise localisation of obstructive lesions. Further uses of the 

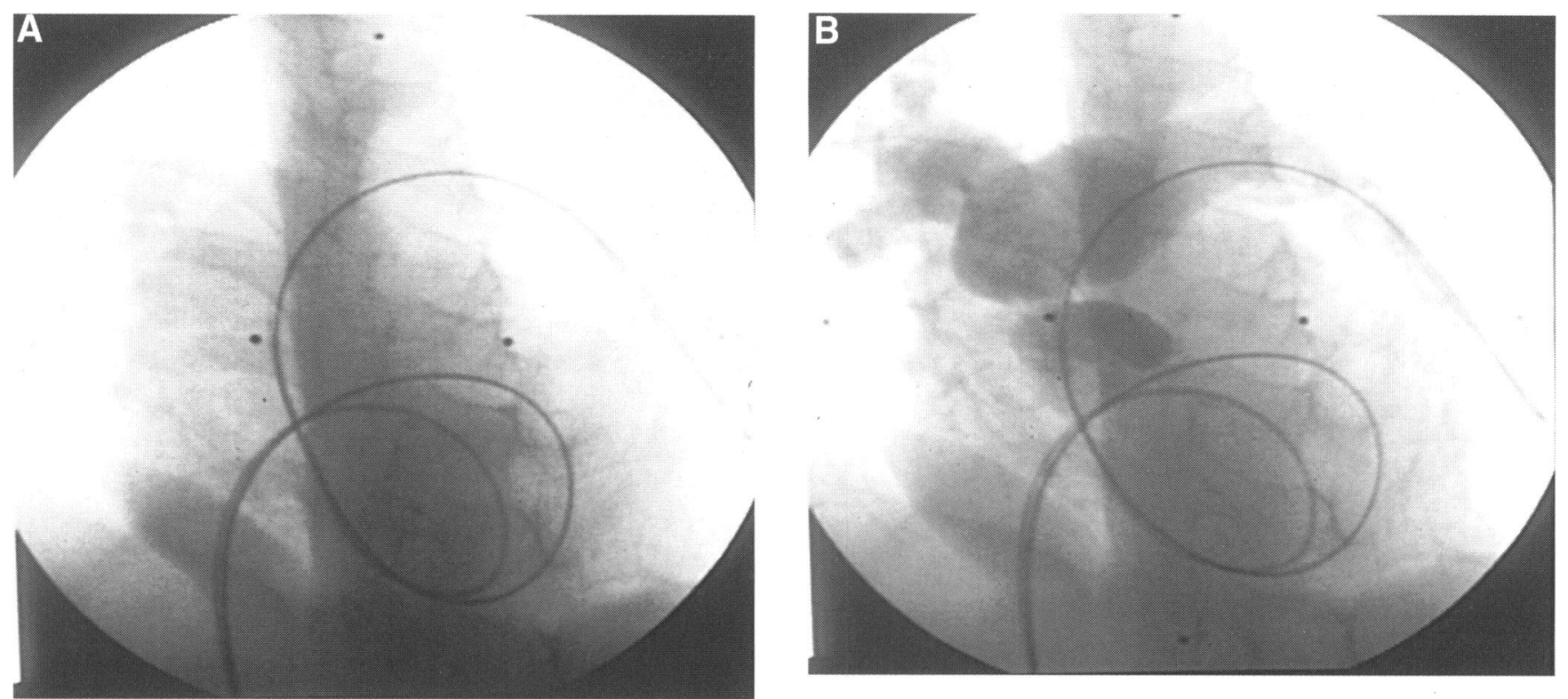

Figure 5 The guidewire is positioned in the left pulmonary branch in a patient with tricuspid atresia with transposition of the great arteries who underwent banding of the pulmonary artery. Despite the complicated route of the wire (inferior vena cava-right atrium-atrial septal defect-left atrium - left ventricle - transposed pulmonary artery) the Multi-Track Angio catheter could be advanced easily into the pulmonary artery (A). The pressure gradient over the banding could be measured by a controlled pullback procedure and angiography could be performed just proximal to the band without catheter recoil $(B)$.

Multi-Track system include multicatheter approaches using similar techniques as those that we used for percutaneous dilatation of the mitral valve. ${ }^{2}$ Future developments of the system will include dedicated mapping and ablation catheters for the use in electrophysiology and in other fields of cardiovascular radiology.

\section{INTERVENTIONAL CATHETERISATION}

In most patients in this series the Multi-Track Angio system was used during interventional cardiac catheterisation. This was because after a standard guidewire had been placed across the lesion to be treated, repeated pressure measurements and angiography could be obtained without any further guidewire manipulation, thereby largely reducing procedure time and limiting potential complications. This allowed us to modify and to simplify even some well established interventional procedures, such as ballon dilatation of the pulmonary valve. ${ }^{89}$ Nonetheless, the major impact of the technique lies in the safe and effective management of stenotic lesions of the great arteries. ${ }^{10-12}$ In particular in patients with re-coarctation of the aorta repeated angiography and pressure measurements can be obtained without the need for guidewire manipulation. This considerably improves the safety of gradual dilatation. Although we did not treat such patients in our series, the MultiTrack system also seems likely to be a great help in angiographic evaluation during implantation of endovascular stents within the pulmonary arteries. Stent delivery, implantation, and angiographic control can all be performed over the same guidewire. At no time would it be necessary to use further vascular access or to withdraw the guidewire to evaluate the final result of the procedure.

SAFETY

No complications were encountered during the use of the Multi-Track system in these $\mathbf{8 4}$ patients. In particular, we did not encounter any episodes of significant blood loss, which can be caused when both the guidewire and the catheter are introduced side-by-side through the haemostatic valve of the introducer sheath. We believe that the use of the Multi-Track Angio catheter reduced the procedure time and the amount of contrast medium needed for detailed angiographic studies, in particular in young infants, ${ }^{12}$ and decreased the risk of angiography and guidewire manipulation within the heart and vessels.

\section{CONCLUSIONS}

The Multi-Track Angio catheter is a new versatile tool that enhances both angiography and haemodynamic studies. Decreased guidewire manipulations and catheter exchanges reduce the procedure time and the potential risk of complication. This is particularly useful in complex diagnostic and interventional cardiac catheterisation in patients with congenital heart disease.

We thank Mr A Tower and Mr M Martin (MAP Medical, North Lawrence, NY 12967) for their invaluable help in developing the Multi-Track Angio catheters. OS is supported by grant from the European Community (Human capital and mobility-ERB CH BGCT 9303 73).

1 Verma R, Keane JF. Use of cutoff pigtail catheters with intraluminal guidewires in interventional procedures in congenital heart disease. Cathet Cardiovasc Diagn 1994;33:85-8.

2 Bonhoeffer P, Piéchaud JF, Sidi D, Yonga G, Jowi C, Joshi $\mathrm{M}$, et al. Mitral dilatation with the Multi-Track system: M, et al. Mitral dilatation with the Multi-Track system: an alternative

3 Tascon PJ, Delgado J, Gomez PC, Albarran GA, Llovet VA, Kabbani Z, et al. New technique for right heart catheterisation using a Mullins' sheath Cathet Cardiovasc Diagn 1993;28:260-2.

4 Jayne JE, Catherwood E, Niles NW, Friedman BJ. Doubleumen catheter assessment of aortic stenosis: comparison with separate catheter technique. Cathet Cardiovasc Diagn 1993;29:157-60.

5 Nakanishi T, Matsumoto $Y$, Seguchi $M$, Nakazawa $M$, Imai $Y$, Momma $\mathrm{K}$. Balloon angioplasty for postoperative pulmonary artery stenosis in transposition of the great arteries. $\mathcal{F}$ Am Coll Cardiol 1993;22:859-66. 
6 Li J, Harrison JK, O’Laughlin MP, Bashore TM, Garson A. Adult patients with congenital heart disease. NC Med f 1994;55:147-8.

7 O'Laughlin MP, Perry SB, Lock JE, Mullins CE. Use of endovascular stents in congenital heart disease. Circulation 1991;83:1923-39.

8 Guarnera S, Piechaud JF, Kachaner J, Le Bidois J, Cohen L, Sidi $\mathrm{D}$, et al. Traitement des stenoses valvulaires pulmidi D, et al. Traitro kulcutanee. Arch Fr Pediatr 1989;46:503-8.

9 Piechaud JF, Ladeia AM, Da Cruz E, Gournay V, Iserin L chaud J , Ladeia AM, Da Cruz E, Gournay V, Iserin L Delogu A, et al. Perforation-dilatation des atresies pulveau-ne et le nourrisson. Arch Mal Coeur 1993;86:581-6.
10 Gentles TL, Lock JE Perry SB. High pressure balloon angioplasty for branch pulmonary artery stenosis: early experience. F Am Coll Cardiol 1992;22:867-72.

11 Edwards BS, Lucas RJ, Lock JE, Edwards JE. Morphologic changes in the pulmonary arteries after percutaneous balloon angioplasty for pulmonary arterial stenosis. Circulation 1985;71:195-201.

$12 \mathrm{De}$ Bono D. Complications of diagnostic cardiac catheterisation: results from 34041 patients in the United Kingdom confidential enquiry into cardiac catheter complications. The Joint Audit Committee of Physicians of London. Br Heart $\mathcal{F}$ 1993;70: $297-300$. 